\title{
Endostar enhances the antitumor effects of radiation by affecting energy metabolism and alleviating the tumor microenvironment in a Lewis lung carcinoma mouse model
}

\author{
YONG-FA ZHENG ${ }^{1}$, WEI GE ${ }^{1}$, HUI-LIN XU ${ }^{1}$, DE-DONG CAO ${ }^{1}$, LIANG LIU ${ }^{1}$, PING-PO MING ${ }^{1}$, \\ CHANG-HU LI ${ }^{1}$, XI-MING XU ${ }^{1}$, WEI-PING TAO ${ }^{1}$ and ZE-ZHANG TAO ${ }^{2}$ \\ Departments of ${ }^{1}$ Oncology and ${ }^{2}$ Otolaryngology, \\ Renmin Hospital of Wuhan University, Wuhan, Hubei 430060, P.R. China
}

Received June 18, 2014; Accepted March 2, 2015

DOI: $10.3892 / 01.2015 .3679$

\begin{abstract}
Lung cancer is a leading cause of morbidity and mortality. Previous studies have identified that an improvement in treatment efficacy was achieved using Endostar; however, the role of Endostar in lung cancer remains poorly understood. The present study investigated whether the enhanced antitumor effects of Endostar in combination with radiation involved changes in the metabolism and microenvironment in non-small cell lung cancer. A Lewis lung carcinoma mouse model was used, including the control, Endostar (ES), radiotherapy (RT) and Endostar plus radiotherapy (ES + RT) groups. The tumor inhibition rates and growth were described based on changes in tumor volume. In addition, ultraviolet enzymatic analysis was performed to determine the lactate level and reverse transcription-polymerase chain reaction was used to measure the mRNA expression of lactate dehydrogenase (LDH). A Meph-3 pH meter was used to detect the ranges of tumor interstitial tissue $\mathrm{pH}$, and immunohistochemical analysis was adopted to examine hypoxia within the tumor microenvironment. The tumor inhibition rate of the ES + RT group was significantly higher compared with the other three groups $(\mathrm{P}<0.05)$. Following treatment, the lactate levels decreased in all three treatment groups compared with the control, particularly in the ES + RT group $(\mathrm{P}<0.05)$. Reduced LDH expression and hypoxic fraction in the tumor microenvironment were also observed in the ES + RT group $(\mathrm{P}<0.05)$. Furthermore, changes from acidic to alkaline $\mathrm{pH}$ in the tumor microenvironment were detected in the ES + RT group. The present study suggested
\end{abstract}

Correspondence to: Professor Ze-Zhang Tao, Department of Otolaryngology, Renmin Hospital of Wuhan University, 238 Jiefang Road, Wuhan, Hubei 430060, P.R. China

E-mail: zezhangtaocn@126.com

Professor Wei Ge, Department of Oncology, Renmin Hospital of Wuhan University, 238 Jiefang Road, Wuhan, Hubei 430060, P.R. China

E-mail: gewei514@126.com

Key words: endostar, radiotherapy, lactate, hypoxia, lung cancer that Endostar is involved in the regulation of metabolism and tumor microenvironment hypoxia, which may be responsible for the enhanced antitumor effect of Endostar in combination with radiotherapy.

\section{Introduction}

Lung cancer is one of the most common causes of cancer-associated mortality worldwide, with a 5-year survival rate of only $\sim 15 \%(1,2)$. Approximately $80 \%$ of lung malignancies are non-small cell lung cancer (NSCLC) (3), and $>50 \%$ of these patients have advanced invasion and/or metastasis, which require further postoperative treatment, including chemotherapy, radiotherapy and biotherapy. Although the prognosis remains poor, significant improvements have been made in the efficacy of lung cancer treatments, with molecular targeted therapy being one of the most efficient therapies (4-7). Previous studies have demonstrated that molecular targeted therapy may enhance the antitumor effect of radiotherapy by inducing apoptosis or inhibiting proliferation of tumor cells $(8,9)$. However, the radiosensitivity effect of molecular targeted therapy remains to be fully elucidated.

Endostar is a recombinant human endostatin, which was approved by the Food and Drugs Administration of China in 2005 for use in the treatment of non-small-cell lung cancer (10). It specifically induces apoptosis and potently inhibits endothelial cell proliferation, angiogenesis and tumor growth $(10,11)$. However, the enhanced antitumor effect of Endostar in combination with radiotherapy is not completely understood.

Hypoxia, a pathophysiological characteristic of solid malignancies, interferes with the fixation of DNA damage and is, therefore, a major cause of resistance to irradiation (12). A previous study of our group revealed that Endostar is involved in the radiosensitivity of lung cancer by inhibiting the expression of hypoxia-inducible factor $1 \alpha$ (HIF-1 $\alpha)(13,14)$. In cancer cells, HIF-1 $\alpha$ induces the expression and enhances the activity of several glycolytic proteins that differ from those detected in nonmalignant cells, including transporters (glucose transporters 1 and 3) and enzymes (hexokinase I and II, and phosphofructokinase-L) (15). Carbonic 
anhydrase IX (CA IX) contributes to the acidification of the tumor environment by efficiently catalyzing the hydration of carbon dioxide to bicarbonate and protons with its extracellularly situated active site. CA IX expression is strongly induced by hypoxia, which is present in numerous tumors and regulated by the transcription factor, $\operatorname{HIF}(16)$.

The present study hypothesized that the antitumor effect of the antigenic agent, Endostar, in combination with radiotherapy was associated with glycolysis and changes of tumor environment $\mathrm{pH}$. In order to investigate the possible mechanism responsible for the enhanced tumor killing effect of Endostar and radiotherapy, changes in the metabolism and hypoxic tumor fraction were determined in a Lewis lung carcinoma (LLC) mouse model. LLC mouse model is a commonly-used model in lung cancer research, since it is easy to produce, with low cost and high rate of tumor formation. This model has favorable conditions for the study of the pathogenesis of lung cancer, drug treatments and biological treatments. To the best of our knowledge, the present study is the first to investigate the combination of Endostar administration and radiotherapy in a LLC mouse model.

\section{Materials and methods}

Tumor model and groups. Inbred C57BL/6 male mice (age, 6-7 weeks; weight, 18-22 g) were purchased from the Experimental Animal Center of Wuhan University (SYXK2003-0013; Wuhan, China). Animals were bred in a barrier-free animal house in the First Clinical College of Wuhan University Laboratory Animal Center (SPFIII; Wuhan, China). This study was performed in strict accordance with the recommendations of the Guide for the Care and Use of Laboratory Animals (8th edition, 2011). The Animal Use Protocol was reviewed and approved by the Institutional Animal Care and Use Committee at Renmin Hospital of Wuhan University.

An LLC model was established by adopting a tumor-bearing tumor cell inoculation in vitro method. LLC tumor cells (purchased from the Chinese Academy of Medical Sciences, Beijing, China) were inoculated in C57BL/6 mice. When a tumor volume of $\sim 0.125 \times 0.125 \times 0.125 \mathrm{~cm}$ was developed on the right shoulder, the mice were sacrificed and the tumor tissue was removed and dispersed into a cell suspension by the enzymatic hydrolysis method. Briefly, tumor tissue was hydrolyzed with $1 \%$ collagenase VI (Sigma-Aldrich, St. Louis, MO, USA), incubated at $37^{\circ}$ for $50 \mathrm{~min}$, pipetted and filtrated. Next, single cell suspensions were collected in centrifuge tubes and centrifuged at $500 \mathrm{xg}$ for $5 \mathrm{~min}$; then, the supernatant was discarded, the cells were resuspended with phosphate-buffered saline (Beyotime Institute of Biotechnology, Shanghai, China), and the cell suspensions were prepared.

Subsequently, C57BL/6 mice were subcutaneously injected with $0.2 \mathrm{ml}$ carcinoma cell suspension $\left(2 \times 10^{6}\right.$ living cells $)$ into the left armpit. When the maximum tumor diameter reached $10 \mathrm{~mm}$ (after 7-10 days), 192 tumor-bearing mice were randomly divided into four groups ( $\mathrm{n}=48$ in each group) as follows: control; Endostar (ES); radiotherapy (RT); and radiotherapy plus Endostar (ES + RT) groups. Six subgroups were formed according to the different time points at which the mice were sacrificed (days 2, 4, 6, 8, 10 and 12). The mice received treatment once per day.
Mice in the control group were subjected to subcutaneous injection of $0.2 \mathrm{ml} 0.9 \%$ normal saline. In the ES group, the mice were subjected to subcutaneous injection of $0.2 \mathrm{ml}$ Endostar $(2 \mathrm{mg} / \mathrm{ml})$. The mice in the radiotherapy group were subjected to subcutaneous injection of $0.2 \mathrm{ml} 0.9 \%$ normal saline on the aforementioned time points (days 2, 4, 6, 8, 10 and 12), followed by $2 \mathrm{~Gy}$ radiation that was topically used on the tumor between days 6 and 10. Mice in the ES + RT group were subjected to subcutaneous injection of $0.2 \mathrm{ml}$ Endostar $(2 \mathrm{mg} / \mathrm{ml})$, followed by 2 Gy radiation that was topically used on the tumor between days 6 and 10 .

Tumor volume. When the tumor model was established (maximum tumor diameter, $\sim 10 \mathrm{~mm}$ ), the tumor length and diameter were determined with a vernier caliper at aforementioned time points (days 0, 2, 4, 6, 8, 10 and 12). The tumors volume was measured prior to treatment in each group. Next, 4 mice from each group were sacrificed and soaked for 3-5 $\mathrm{min}$ in $75 \%$ ethanol; then, the right shoulder was cut, and the tumor was removed and washed twice with saline. The tumor tissue samples were stored at $-80^{\circ} \mathrm{C}$ for follow-up experiments.

Tumors in different groups and subgroups were separated following the treatment termination. Tumor volumes were calculated according to the formula $V=a \times b^{2} \times 0.52$, where $a$ is the longest diameter and $b$ is the maximum transverse diameter. Subsequently, growth curves were constructed using Microsoft Office Excel 2010 (Microsoft Corporation, Redmond, WA, USA) and SPSS 16.0 software (SPSS, Inc., Chicago, IL, USA).

Ultraviolet (UV) enzymatic analysis. The tumor tissue samples were ground, homogenized in cold $\mathrm{HClO}_{4}\left(4^{\circ} \mathrm{C}\right)$ and centrifuge at $500 \mathrm{x} \mathrm{g}$ for $10 \mathrm{~min}$. Next, the tissue supernatant was used to measure lactate levels with a UV spectrophotometer (UV-2450/2550; Shimadzu Corporation, Tokyo, Japan). The lactate levels were calculated using the optical density values at $340 \mathrm{~nm}$, which required the use of nicotinamide adenine dinucleotide, L-lactate dehydrogenase (L-LDH) and alanine transaminase (Sigma-Aldrich).

Reverse transcription-polymerase chain reaction (RT-PCR). Total RNAs were isolated from the tumor tissues using TRIzol ${ }^{\circledR}$ reagent (Life Technologies, Grand Island, NY, USA), extracted using chloroform and precipitated using ice-cold isopropanol. Next, cDNA was synthesized from $\sim 1 \mu \mathrm{g}$ mRNA using the ReverTra Ace qPCR RT kit (Toyobo Corporation, Osaka, Japan) according to the manufacturer's instructions. The primer sequences used for PCR were as follows: $\beta$-actin forward, 5'-CACGATGGAGGGGCCGGACTCATC-3', and reverse, 5'-TAAAGACCTCTATGCCAACACAGT-3'; and LDH forward, 5'-TGGCAGCCTCTTCCTTAAAA-3', and reverse, 5'-CAGCTTGCAGTGTGGACTGT-3'. Quantitative RT-PCR was performed using the Thunderbird $\mathrm{SYBR}^{\circledR} \mathrm{qPCR}$ Mix (QPS201, QPS-201T; Toyobo Corporation). All the reactions were prepared in $10 \mathrm{ml}$ samples using the standard PCR conditions according to the manufacturer's instructions. $\beta$-actin was used as a control.

$p H$. The mice were anesthetized by intraperitoneal injection of $0.15 \mathrm{ml} 1.5 \%$ sodium pentobarbital (Sigma-Aldrich). Next, a $1.0 \mathrm{~cm}$ incision was made in the tumor. A pH microelectrode 


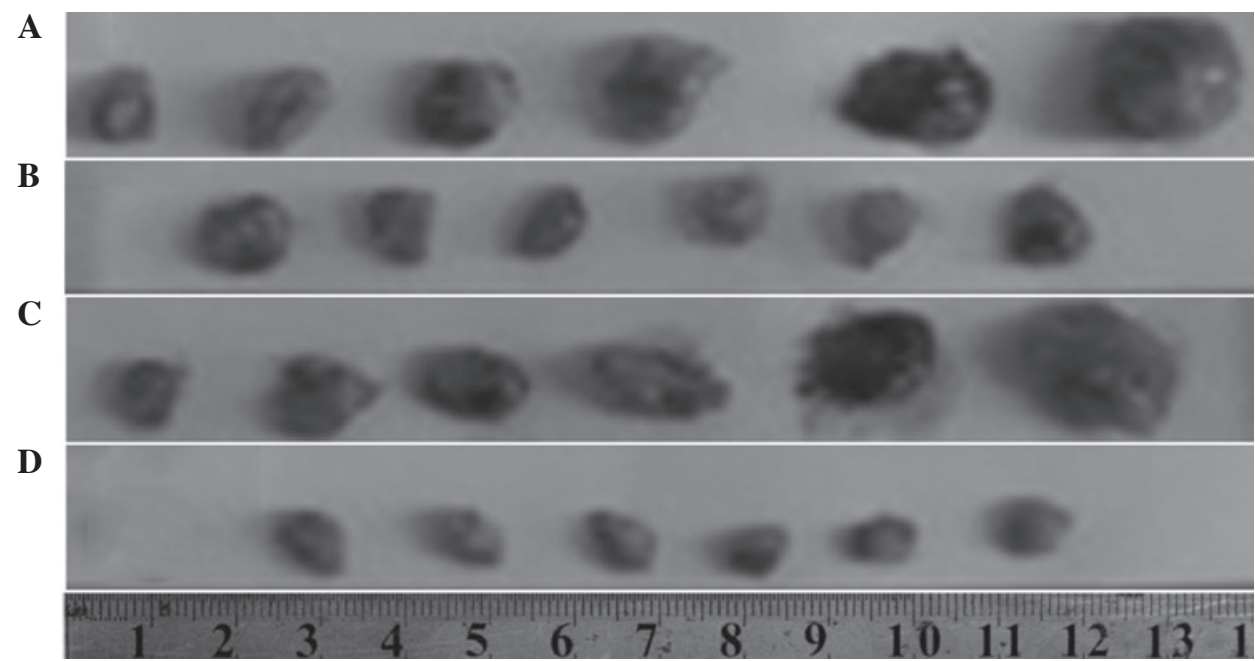

Figure 1. Tumor samples of Lewis lung carcinoma samples from the four different groups between days 2 and 12. (A) Control; (B) Endostar; (C) radiotherapy and (D) Endostar + radiotherapy groups.

A

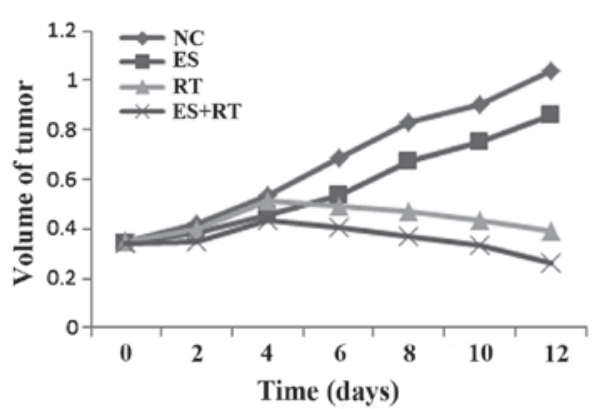

C

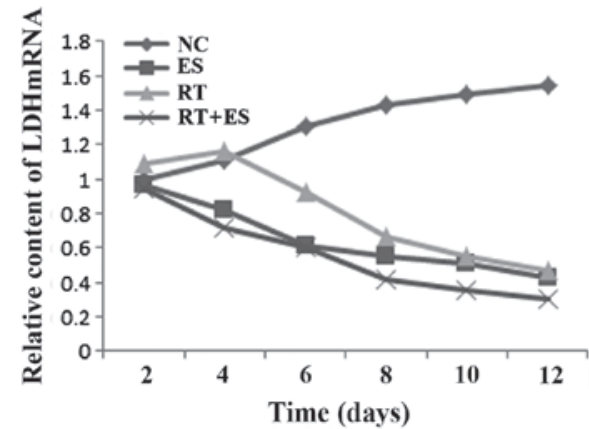

B

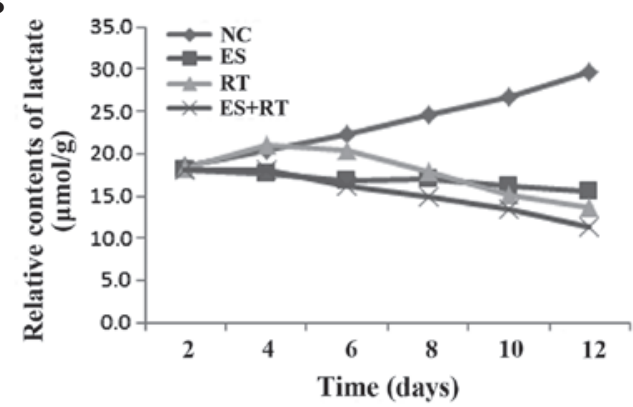

D

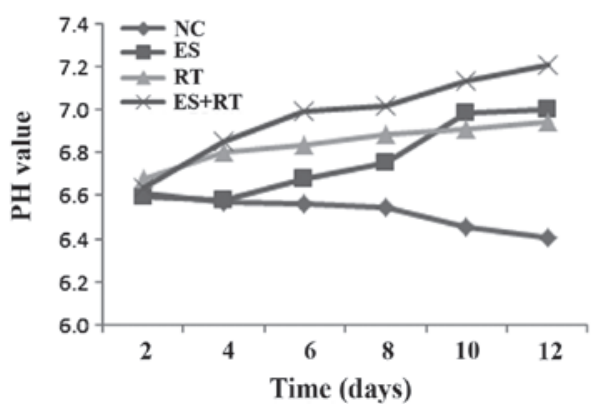

Figure 2. Changes in the tumor volumes, $\mathrm{LDH}$ levels and $\mathrm{pH}$ following treatment. (A) Growth curve of tumor in the Lewis lung adenocarcinoma mouse model. Tumor volumes were measured following separation of tumors. (B) Changes of lactate levels in the tumors, which were detected between day 2 and day 12 in all the groups. (C) LDH mRNA levels in tumor tissues on different days. Tumor samples were prepared and kept in $-80^{\circ} \mathrm{C}$. The expression of lactate mRNA was measured using reverse transcription-polymerase chain reaction between day 0 and day 12. (D) pH values of tumor tissues on different days. Groups: NC, normal control; RT, radiotherapy; ES, Endostar; ES + RT, Endostar + radiotherapy. LDH, lactate dehydrogenase.

(Meph-3 pH meter, PH-016; Shanghai Sunlight Opto Device Co., Ltd., Shanghai,China) was inserted $4.0 \mathrm{~mm}$ into the tumor tissue in order to detect the $\mathrm{pH}$.

Immunohistochemical analysis. Hypoxyprobe ${ }^{\mathrm{TM}}-1$ kit (Hypoxyprobe, Inc., Burlington, MA, USA), containing the anti-pimonidazole mouse immunoglobulin G1 monoclonal antibody, was used to detect tumor hypoxia. Hematoxylin and eosin, as well as substance $\mathrm{P}$, were used for immunohistochemical analysis. Hypoxia in tumors was detected through the formation of pimonidazole adducts (11) and the Olympus CX21 microscope (Olympus Corporation, Tokyo, Japan) was used to identify the hypoxic tumor cells. Next, pimonidazole hydrochloride was intraperitoneally injected at a dose of $60 \mathrm{mg} / \mathrm{kg}$. At $1 \mathrm{~h}$ after injection, the mice were sacrificed, and the tumor tissue was prepared and detected following the manufacturer's instructions.

Statistical analysis. All the results are expressed as the mean \pm standard error of mean and were analyzed using SPSS 16.0 software (SPSS, Inc., Chicago, IL, USA). The $F$-test was applied to assess differences between the groups. 

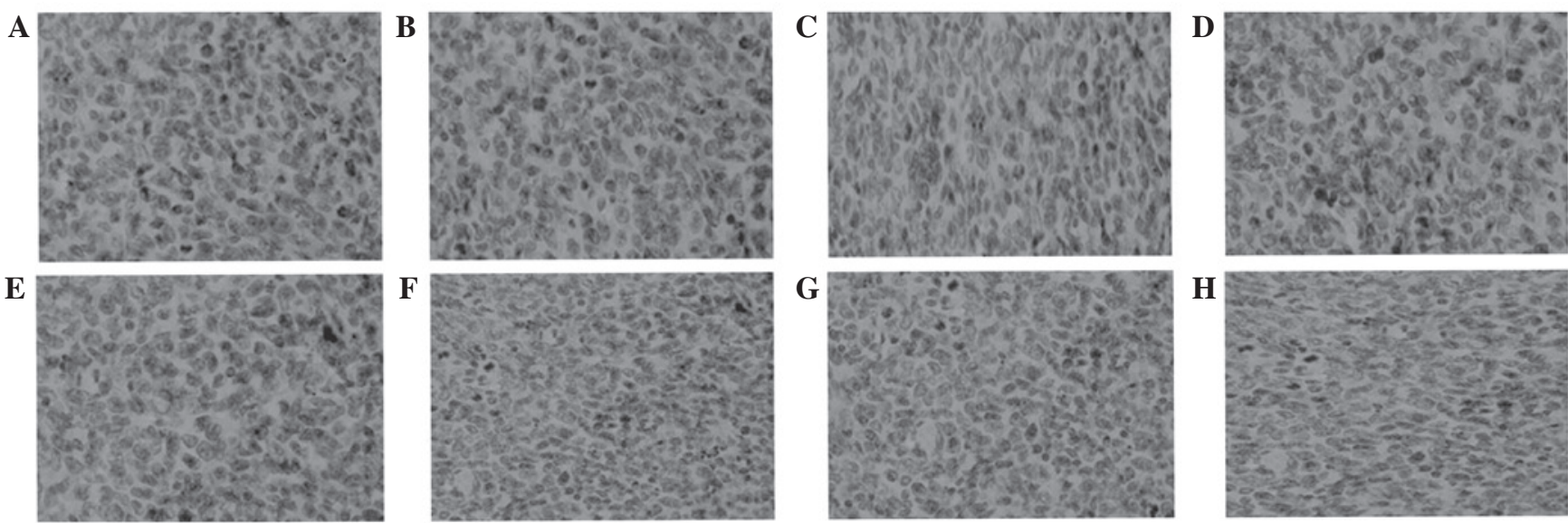

Figure 3. Immunohistochemical analysis of hypoxic cell samples collected (A-D) at day 10 and (E-H) at day 12, in each group (hematoxylin and eosin stain; magnification, x400). Groups: (A and E) normal control; (B and F), radiotherapy; (C and G) Endostar; (D and H) Endostar + radiotherapy.

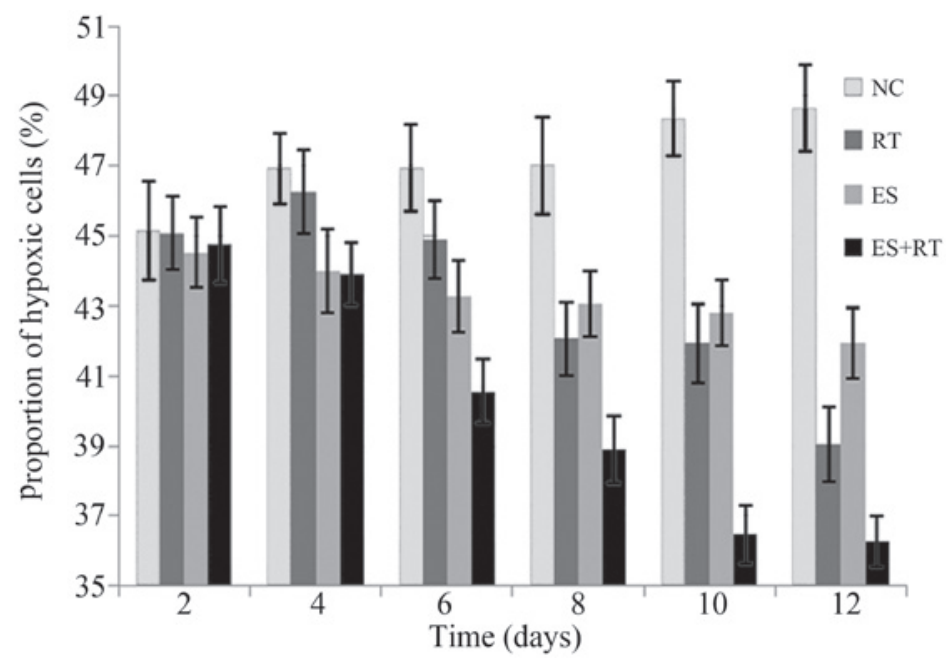

Figure 4. Proportion of hypoxic cells following treatment with Endostar and/or radiation. The hypoxic cells were marked using immunohistochemistry and the percentage of hypoxic cells was observed under a microscope. Groups: NC, normal control; RT, radiotherapy; ES, Endostar; ES + RT, Endostar + radiotherapy.

In all the statistical analyses, $\mathrm{P}<0.05$ was considered to indicate a statistically significant difference.

\section{Results}

Tumor growth. Compared with the control group, treatment with Endostar alone was found to only slightly inhibit the tumor proliferation (ES group; $\mathrm{P}>0.05$ ). However, treatment with Endostar in combination with 2 Gy radiation significantly suppressed increases in tumor volume between days 6 and 12 $(\mathrm{ES}+\mathrm{RT}$ group; $\mathrm{P}<0.05)$. Differences in tumor growth inhibition between the ES + RT and RT groups were also statistically significant between days 6 and $10(\mathrm{P}<0.05)$. These results indicated that the ES + RT group exhibited increased tumor growth inhibition when compared with the other three groups (Fig. 1).

Lactate levels. Lactate is considered a dead-end product of glycolysis, and its generation and accumulation promote tumor growth and metastasis. With the recent advances in tumor metabolism and gene therapy, lactate was identified as a potential therapeutic target in tumors (17-20). Tumor cells can also uptake and utilize lactate, and a high concentration of lactate is a sign or marker of tumor metabolic adaptation, suggesting a poor prognosis (17-20).

As shown in Fig. 2, the lactate levels in the ES, RT and ES+RT groups were significantly lower compared with the control group between days 6 and $10(\mathrm{P}<0.05)$. In addition, the lactate level in the ES + RT group was reduced the most, when compared with the ES and RT groups $(\mathrm{P}<0.05)$. The decreased lactate levels show that the treatments reduced tumor metabolism and inhibited tumor growth.

LDH mRNA. As shown in Fig. 2C, the LDH mRNA expression in each group significantly decreased between days 6 and 10, when compared with the control group $(\mathrm{P}<0.05)$. The expression of LDH mRNA in the ES + RT group was lower compared with the ES and RT groups, and the difference was statistically significant $(\mathrm{P}<0.05)$. These results show that the RT, ES and $\mathrm{ES}+\mathrm{RT}$ treatments reduced the in vivo expression levels of LDH in the tumor tissues. Thus, ES+RT treatment with radiotherapy may exhibit a synergistic effect in the regulation of tumor metabolism. 
$p H$. All the groups exhibited an increasing trend in the tumor $\mathrm{pH}$ values between days 6 and 10, when compared with the control group $(\mathrm{P}<0.05)$. As shown in Fig. 4, the changes in $\mathrm{pH}$ between days 6 and 10 also indicated that the ES + RT group presented a significantly increased $\mathrm{pH}$ when compared with the ES and RT groups $(\mathrm{P}<0.001)$. The increased $\mathrm{pH}$ value shows that the treatments reduced the acidification of the tumors.

Hypoxic cell fraction. Following immunohistochemical analysis, tumor cells exhibiting brown particles in the cytoplasm were identified as hypoxic tumor cells and assessed under a microscope (Figs. 3 and 4). The results indicated that the hypoxic cell fractions in the treatment groups were significantly decreased when compared with the control group after five days of treatment $(\mathrm{P}<0.05)$, particularly in the ES+RT group. The hypoxic cell fraction in the ES+RT group was markedly lower compared with the ES and RT groups, and the difference was statistically significant $(\mathrm{P}<0.001)$. The decreased hypoxic cell fraction indicates that the treatments reduced hypoxia in the tumor micro-environment.

\section{Discussion}

The cellular metabolism of lung cancer tissues is markedly different from that of normal tissues. Major metabolic changes are known as 'aerobic glycolysis', and are accompanied with hypoxia and an acidulated tumor microenvironment $(21,22)$. Micro-environmental hypoxia in tumor cells is one of the causes of resistance to chemotherapy and/or radiation in solid tumors (23-25). For instance, hypoxia induces the expression of multidrug resistance gene 1 in the tumor, increasing the resistance of tumor cells to chemotherapeutic drugs. In addition, hypoxia and HIF-1 are able to induce the expression of various tumor genes, resulting in tumor cells tolerance to radiotherapy. Consequently, the antitumor effects of radiotherapy and chemotherapy may be hampered (23-25). In the present study, an LLC mouse model was used to investigate the effect of Endostar treatment and radiotherapy. The results indicated that Endostar may enhance the efficacy of radiation by reducing hypoxia and acidification in the tumor microenvironment, finally resulting in suppression of tumor growth.

Treatment with radiation causes generation of reactive oxygen species, including superoxide radical anions and hydroxyl radicals $(26,27)$. Thus, an accumulation of antioxidants, such as lactate, may induce or enhance resistance to radiation (28). The results of a previous study that included $>1,000$ individual xenografts of human head and neck cancer demonstrated that lactate concentrations are positively correlated with radioresistance (29). This lactate-associated radioresistance was hypoxia-independent, indicating that well-oxygenated high-lactate tumors are radioresistant (30). Tumor cells ensure sufficient oxygen and nutrient supply for proliferation through lactate-induced secretion of vascular endothelial growth factor (VEGF), which results in the formation of new vessels (31).

In the present study, the levels of lactate and LDH mRNA in the ES+RT group were significantly decreased compared with the levels in the RT group between days 6 and 10. This result indicated that Endostar treatment combined with radiotherapy exhibited a synergistic effect on glycolysis inhibition. Therefore, Endostar may suppress glycolysis, leading to reduced lactate production and thereby increased radiosensitivity of the tumor. In addition, the reduced level of lactate may be responsible for the decreased expression of VEGF (32).

An acidic microenvironment (decreased $\mathrm{pH}$ ) may also result from overgeneration of lactate. Cancer cells produce a large amount of lactic acid, which is generated through glucose metabolism and inefficient vascular clearing, resulting in an acidic microenvironment within solid tumors (33). In tumor cells, the lactate generated by glycolysis and the carbonic acid catalyzed by CA IX are the major sources of hydrogen ions $\left(\mathrm{H}^{+}\right)$in the extracellular fluid, reducing the tumor extracellular $\mathrm{pH}$. In the present study, the $\mathrm{pH}$ value in the ES + RT group was significantly improved when compared with the RT and ES groups between days 6 and 10. This observation is consistent with the changes in the lactate levels. Therefore, the status of acidic environment is hypothesized to be positively associated with glycolysis activity in tumor.

Hypoxic tumor cells are more resistant to radiotherapy as a consequence of the interference of hypoxia with the fixation of free radical-induced DNA damage (6). In the present study, immunohistochemical analysis to detect tumor hypoxia indicated that the hypoxic cell fraction in the ES+RT group was significantly decreased after day 6 . This observation is attributed to the continuous intervention of anti-angiogenic agents, which weaken glycolysis and improve the acidic microenvironment. These changes possibly increased the temporary blood and oxygen supplies to meet the increased tumor cell metabolism, thereby indirectly increasing the sensitivity of hypoxic cells to radiation.

Since Jain first proposed the normalization of tumor vasculature (34), several studies on Endostar in chemotherapy and radiotherapy sensitivity have been conducted, which determined the 'normalization window' (35-37). $\mathrm{Rh}$-endostatin may normalize the tumor vasculature and microenvironment in LLCs, possibly through modulation of the balance between VEGF-A and thrombospondin-1 (38). During vascular normalization, treatment with paclitaxel was identified to exhibit a maximal effect on tumor growth inhibition (38). In addition, Endostar was demonstrated to normalize tumor vasculature, which alleviated hypoxia and significantly sensitized the antitumor function of radiation in human nasopharyngeal cancer (39). Based on energy metabolism, the present study investigated the underlying mechanism through which Endostar exhibits a radiosensitization effect. Further studies are required to determine whether normalization of tumor vasculature is associated with changes in the metabolism and microenvironment of tumors.

In conclusion, Endostar may enhance the antitumor effect of radiation by reducing glycolysis, hypoxia and acidification of the tumor microenvironment. These results provide an important experimental basis for the combination of Endostar with radiotherapy in the treatment of lung cancer.

\section{Acknowledgements}

This study was supported by grants from the Key Program of National Natural Science Foundation of China 
(nos. 30970860 and 81272500) and the China International Medical Foundation (no. CIMF-F-H001-001).

\section{References}

1. Siegel R, Naishadham D and Jemal A: Cancer statistics, 2013. CA Cancer J Clin 63: 11-30, 2013.

2. Jemal A, Tiwari RC, Murray T, Ghafoor A, et al: Cancer statistics, 2004. CA Cancer J Clin 54: 8-29, 2004.

3. No authors listed: Cancer facts and figures, 2004. American Cancer Society, Atlanta, GA, 2004.

4. Reungwetwattana T, Weroha SJ and Molina JR: Oncogenic pathways, molecularly targeted therapies, and highlighted clinical trials in non-small-cell lung cancer (NSCLC). Clin Lung Cancer 13: 252-266, 2012.

5. Kobayashi K and Hagiwara K: Epidermal growth factor receptor (EGFR) mutation and personalized therapy in advanced nonsmall cell lung cancer (NSCLC). Target Oncol 8: 27-33, 2013.

6. Bria E, Bonomi M, Pilotto S, et al: Clinical meta-analyses of targeted therapies in adenocarcinoma. Target Oncol 8: 35-45, 2013.

7. Ge W, Cao DD, Wang HM, Jie FF, Zheng YF and Chen Y: Endostar combined with chemotherapy versus chemotherapy alone for advanced NSCLCs: a meta-analysis. Asian Pac J Cancer Prev 12: 2705-2711, 2011.

8. Du Y, Peyser ND and Grandis JR: Integration of molecular targeted therapy with radiation in head and neck cancer. Pharmacol Ther 142: 88-98, 2014

9. Koh PK, Faivre-Finn C, Blackhall FH and De Ruysscher D Targeted agents in non-small cell lung cancer (NSCLC): clinical developments and rationale for the combination with thoracic radiotherapy. Cancer Treat Rev 38: 626-640, 2012.

10. Folkman J: Antiangiogenesis in cancer therapy - endostatin and its mechanisms of action. Exp Cell Res 312: 594-607, 2006.

11. O'Reilly MS, Boehm T, Shing Y, et al: Endostatin: an endogenous inhibitor of angiogenesis and tumor growth. Cell 88: 277-285, 1997.

12. Meijer TW, Kaanders JH, Span PN and Bussink J: Targeting hypoxia, HIF-1, and tumor glucose metabolism to improve radiotherapy efficacy. Clin Cancer Res 18: 5585-5594, 2012.

13. Zhang L, Ge W, Hu K, et al: Endostar down-regulates HIF-1 and VEGF expression and enhances the radioresponse to human lung adenocarcinoma cancer cells. Mol Biol Rep 39: 89-95, 2012.

14. Ge W,Zheng Y,Zhang L, et al: Endostar enhances the radioresponse on Lewis lung carcinoma by regulating HIF-1 $\alpha$. In: Biomedical Engineering and Informatics (BMEI), 2011. 4th International Conference. Ding YS, Peng YH, Shi R, et al (eds). Volume 3. IEEE, Shanghai, pp 1486-1490, 2011

15. Marín-Hernández A, Gallardo-Pérez JC, Ralph SJ, Rodríguez-Enríquez S and Moreno-Sánchez R: HIF-1alpha modulates energy metabolism in cancer cells by inducing over-expression of specific glycolytic isoforms. Mini Rev Med Chem 9: 1084-1101, 2009.

16. Winum JY, Rami M, Scozzafava A, Montero JL and Supuran C: Carbonic anhydrase IX: a new druggable target for the design of antitumor agents. Med Res Rev 28: 445-463, 2008.

17. Yokota H, Guo J, Matoba M, et al: Lactate, choline, and creatine levels measured by vitro $1 \mathrm{H}-\mathrm{MRS}$ as prognostic parameters in patients with non-small-cell lung cancer. J Magn Reson Imaging 25: 992-999, 2007.

18. Bonuccelli G, Tsirigos A, Whitaker-Menezes D, et al: Ketones and lactate 'fuel' tumor growth and metastasis: Evidence that epithelial cancer cells use oxidative mitochondrial metabolism. Cell Cycle 9: 3506-3514, 2010

19. Goetze K, Walenta S, Ksiazkiewicz M, et al: Lactate enhances motility of tumor cells and inhibits monocyte migration and cytokine release. Int J Oncol 39: 453-463, 2011.
20. Yaligar J, Thakur SB, Bokacheva L, et al: Lactate MRSI and DCE MRI as surrogate markers of prostate tumor aggressiveness. NMR Biomed 25: 113-122, 2012.

21. Warburg O: On respiratory impairment in cancer cells Science 124: 269-270, 1956.

22. Denko NC: Hypoxia, HIF1 and glucose metabolism in the solid tumour. Nat Rev Cancer 8: 705-713, 2008.

23. Comerford KM, Wallace TJ, Karhausen J, Louis NA, Montalto MC and Colgan SP: Hypoxia-inducible factor-1 dependent regulation of the multidrug resistance (MDR1) gene. Cancer Res 62: 3387-3394, 2002.

24. Semenza GL: Targeting HIF-1 for cancer therapy. Nat Rev Cancer 3: 721-732, 2003

25. Williams KJ, Telfer BA, Xenaki D, et al: Enhanced response to radiotherapy in tumours deficient in the function of hypoxia inducible factor-1. Radiother Oncol 75: 89-98, 2005.

26. Masaki H, Okano Y and Sakurai H: Generation of active oxygen species from advanced glycation end-products (AGEs) during ultraviolet light A (UVA) irradiation and a possible mechanism for cell damaging. Biochim Biophys Acta 1428: 45-56, 1999.

27. Jagetia GC, Shetty PC and Vidyasagar MS: Inhibition of radiation-induced DNA damage by jamun, Syzygium cumini, in the cultured splenocytes of mice exposed to different doses of $\gamma$-radiation. Integr Cancer Ther 11: 141-153, 2012.

28. Sattler UG and Mueller-Klieser W: The anti-oxidant capacity of tumour glycolysis. Int J Radiat Biol 85: 963-971, 2009.

29. Sattler UG, Meyer SS, Quennet V, et al: Glycolytic metabolism and tumour response to fractionated irradiation. Radiother Oncol 94: 102-109, 2010.

30. Quennet V, Yaromina A, Zips D, et al: Tumor lactate content predicts for response to fractionated irradiation of human squamous cell carcinomas in nude mice. Radiother Oncol 81: 130-135, 2006.

31. Hirschhaeuser F, Sattler UG and Mueller-Klieser W: Lactate: a metabolic key player in cancer. Cancer Res 71: 6921-6925, 2011.

32. Kwasiborski PJ, Kowalczyk P, Mrówka P, et al: Selected, biochemical markers of hypoxia. Przegl Lek 69: 115-119, 2012 (In Polish).

33. Gatenby RA and Gillies RJ: Why do cancers have high aerobic glycolysis? Nat Rev Cancer 4: 891-899, 2004.

34. Jain RK: Normalization of tumor vasculature: An emerging concept of anti-angiogenic therapy. Science 307: 58-62, 2005.

35. Wen QL, Meng MB, Yang B, et al: Endostar, a recombined humanized endostatin, enhances the radioresponse for human nasopharyngeal carcinoma and human lung adenocarcinoma xenografts in mice. Cancer Sci 100: 1510-1519, 2009.

36. Du H, Ge W, Li C, Zhao Z, Xu X and Yang F: Effects of rh-endostar in combination with radiotherapy on rats with lung cancer. Zhongguo Fei Ai Za Zhi 13: 386-390, 2010 (In Chinese).

37. Xu M, Huang H, Xiong Y, Peng B, et al: Combined chemotherapy plus endostar with sequential stereotactic radiotherapy as salvage treatment for recurrent esophageal cancer with severe dyspnea: A case report and review of the literature. Oncol Lett 8: 291-294, 2014.

38. Huang $G$ and Chen L: Recombinant human endostatin improves anti-tumor efficacy of paclitaxel by normalizing tumor vasculature in Lewis lung carcinoma. J Cancer Res Clin Oncol 136: 1201-1211, 2010

39. Peng F, Xu Z, Wang J, et al: Recombinant human endostatin normalizes tumor vasculature and enhances radiation response in xenografted human nasopharyngeal carcinoma models. PLoS One 7: e34646, 2012. 